\title{
Telesimulación en la formación en medicina perioperatoria desde la perspectiva colombiana
}

\author{
Telesimulation in perioperative medicine \\ training from a Colombian perspective
}

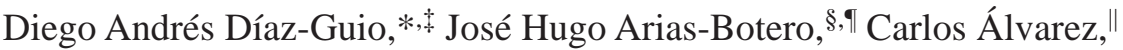 \\ María Helena Gaitán-Buitrago, ${ }^{*, * *, \ddagger}$ Alejandra Ricardo-Zapata,* Lina Cárdenas, ${ }^{*, \ddagger}$

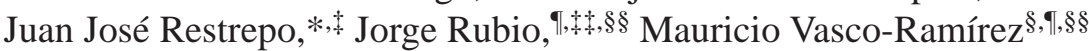

\section{Palabras clave: Simulación clínica, aprendizaje, anestesiología, habilidades no} técnicas, COVID-19.

\section{Keywords: Simulation training, learning, anesthesiology, non-technical skills,}

COVID-19.

* Grupo de Investigación y Simulación Clínica (EdSiMc), Centro de Simulación Clínica

VitalCare, Armenia, Colombia.

‡ Facultad de Medicina, Universidad "Alexander Von Humboldt",

Armenia, Colombia.

$\S$ Facultad de Medicina, Universidad CES,

Medellín, Colombia.

" Sociedad Antioqueña de Anestesiología y Reanimación. SADEA, Medellín, Colombia.

\| Facultad de ciencias

de la salud, Universidad Cooperativa de

Colombia, Medellín, Colombia.

** Doctorado

en Ciencias de

la Educación,

RUDECOLOMBIA -

Universidad del Quindío,

Armenia, Colombia.

\# Salud SURA,

Medellín, Colombia.

$\S \S$ Sociedad Colombiana

de Anestesiología y

Reanimación. S.C.A.R.E,

Bogotá, Colombia.

\section{RESUMEN}

La pandemia por SARS-CoV-2 ha presionado una acelerada virtualización de la educación con el apoyo de tecnologías de la información y la comunicación. En ciencias de la salud se ha vuelto rutinario el uso de conferencias en línea tanto en la formación de pregrado como en la especializada; sin embargo, esta masificación ha traído consigo en gran medida desmotivación en los estudiantes. La simulación clínica presencial ha demostrado ser de utilidad en los procesos de enseñanza-aprendizaje al ser una práctica social que permite la construcción de aprendizaje a través de la experiencia y la reflexión consciente e intencionada. En 2020 se cerraron los centros de simulación dejando un vacío evidente, como respuesta a esta necesidad la simulación no presencial se ha tornado en una opción interesante en la formación en ciencias de la salud en general y en medicina perioperatoria en particular, por lo tanto, como educadores debemos reflexionar sobre sus fortalezas, debilidades y posibles aplicaciones.

\section{ABSTRACT}

SARS-CoV-2 pandemic has driven an accelerated virtualization of education with the support of information and communication technologies. In the health sciences, the use of online conferencing in both university and specialized training has become routine, nonetheless, this has largely demotivated students. Face-to-face clinical simulation has proven useful in teaching-learning processes as it is a social practice that allows the construction of learning through experience and conscious and intentional reflection. In 2020 the simulation centers were closed, leaving an obvious void, as a response to this need, non-presential simulation has become an interesting option in training in health sciences in general and in perioperative medicine in particular, therefore, as educators we must reflect on its strengths, weaknesses, and possible applications.

\section{INTRODUCCIÓN}

T a simulación clínica es una metodología de Lenseñanza y aprendizaje bien posicionada en la educación en pregrado, postgrado y educación médica continuada en medicina perioperatoria..$^{1-3}$ La simulación clínica como práctica social ${ }^{4}$ constituye una mediación educativa entre el aula de clase y las áreas de práctica clínica que busca representar la realidad, ${ }^{5}$ permitiendo así el aprendizaje de conceptos, ${ }^{6}$ el desarrollo de habilidades motoras y de habilidades no técnicas ${ }^{7,8}$ sin poner en riesgo la integridad de los pacientes ni la seguridad psicológica de quienes aprenden.

La pandemia por coronavirus (COVID-19) ${ }^{9}$ ha obligado a la población mundial a mantener un distanciamiento social, esto incluye al mundo académico. ${ }^{10}$ Las universidades y centros de formación durante 2020 y parte de 2021 cerraron sus aulas y empezaron a utilizar estrategias en entornos virtuales, apoyadas por las tecnologías de la comunicación y la información. ${ }^{11}$

La educación basada en conferencias en línea (webinar) ha sido una de las estrategias más

Citar como: Díaz-Guio DA, Arias-Botero JH, Álvarez C, Gaitán-Buitrago MH, Ricardo-Zapata A, Cárdenas L, et al. Telesimulación en la formación en medicina perioperatoria desde la perspectiva colombiana. Rev Latinoam Simul Clin. 2021; 3 (3): 110-116. https://dx.doi.org/10.35366/103187 
Recibido: 07/09/2021 Aceptado: 15/11/2021

doi: $10.35366 / 103187$ usadas, permite revisar conceptos y posiblemente construir conocimientos declarativos; no obstante, presenta limitaciones al ser una modalidad centrada en la enseñanza, tiende a ser pasiva y puede menoscabar la motivación de los estudiantes para aprender. Una opción interesante para mantener los procesos de enseñanza-aprendizaje en estos tiempos difíciles es la simulación clínica no presencial, metodología que se ha desarrollado en la última década; ${ }^{12-14}$ esta estrategia ha mostrado ser de utilidad para el aprendizaje de conceptos y la satisfacción de los estudiantes.

En este artículo inicialmente reflexionaremos sobre el papel de la simulación clínica no presencial en la formación del equipo de salud en medicina perioperatoria (atención multidisciplinaria, centrada en el paciente, que ocurre durante todo el proceso quirúrgico hasta su recuperación), ${ }^{15,16}$ fundamentándonos en la literatura y en nuestra experiencia en su uso como educadores especializados y expertos clínicos. Además, exponemos la manera en que aplicamos esta metodología a partir del diseño instruccional, lo que puede ser de gran utilidad para el lector que quiera iniciarse o mejorar en su uso.

\section{NECESIDADES DE APRENDIZAJE EN LA PANDEMIA}

Como a muchos profesionales de la salud, la pandemia por SARS-CoV-2 nos ha planteado la necesidad de formación en diferentes áreas del conocimiento para responder a la emergencia y adaptarnos a esta nueva normalidad, ${ }^{17,18}$ entre las que se encuentran las pautas de bioseguridad en la unidad de cuidados intensivos y áreas de expansión, ${ }^{19}$ el uso seguro de equipo de protección individual (EPP), ${ }^{20}$ uso de ventiladores mecánicos y entrenamiento en factores humanos. ${ }^{21}$

En medicina perioperatoria, estas necesidades antes de la pandemia eran cubiertas a través de la simulación clínica de manera presencial ${ }^{22}$ en los centros de simulación o en las áreas de práctica clínica; sin embargo, ante la limitación generada por el contexto y la necesidad de ofrecer seguridad a estudiantes de pregrado y postgrado, a profesionales de la salud en programas de desarrollo permanente y al personal docente, comenzamos a buscar alternativas de formación a través de un formato similar al que ya usábamos en los centros de simulación, con ayuda de las tecnologías de la información y la comunicación dentro de un marco de escepticismo racional.

\section{SIMULACIÓN CLÍNICANO PRESENCIAL, EL PROBLEMA CON LA TERMINOLOGÍA}

Antes de adentrarnos en el dominio de un nuevo concepto, merece la pena orientar el contexto "tradicional" de la simulación presencial. Entendemos la simulación clínica de alta fidelidad estructurada como una actividad social por excelencia ${ }^{4}$ en la que las personas interactúan entre sí, con simuladores y con biotecnología en un entorno seguro de aprendizaje que se asemeja a los escenarios de práctica cotidiana, para luego hacer

Tabla 1: Términos usados en la simulación no presencial. ${ }^{14,23,25,26}$

\begin{tabular}{|c|c|c|}
\hline Concepto & Significado & Utilidad \\
\hline Simulación remota & $\begin{array}{l}\text { Uno o varios operadores pueden manejar un simulador que sea controlado por una computadora } \\
\text { personal de manera no presencial a través de internet. } \\
\text { El facilitador/instructor no está presencialmente en el sitio, los participantes y el simulador sí están. } \\
\text { El facilitador/instructor orienta la sesión, evalúa el desempeño y realiza la reflexión y/o retroalimentación } \\
\text { a través de alguna plataforma de conferencias. }\end{array}$ & $\begin{array}{l}\text { enseñanza } \\
\text { evaluación }\end{array}$ \\
\hline Simulación en línea & $\begin{array}{l}\text { Diferentes actividades de aprendizaje que se simulan a través de la pantalla de una computadora } \\
\text { conectada a internet. } \\
\text { Puede ser asincrónica (en diferido) como en la videosimulación o sincrónica (en tiempo real) } \\
\text { como la telepresencia. } \\
\text { La simulación sincrónica permite la introducción, simulación y debriefing en tiempo real. }\end{array}$ & $\begin{array}{l}\text { enseñanza } \\
\text { aprendizaje } \\
\text { evaluación }\end{array}$ \\
\hline Telesimulación & $\begin{array}{l}\text { Uso de componentes de la simulación junto a tecnologías de la comunicación para enseñar, } \\
\text { entrenar y evaluar. } \\
\text { Los participantes están en sitios diferentes a los instructores, realizan simulación a través de una } \\
\text { computadora conectada a internet. } \\
\text { Permite la introducción, simulación y debriefing en tiempo real. }\end{array}$ & $\begin{array}{l}\text { enseñanza } \\
\text { entrenamiento } \\
\text { evaluación }\end{array}$ \\
\hline
\end{tabular}


una reunión dialéctica e intencionada (debriefing) donde se reflexiona sobre las actuaciones propias y del equipo, con el fin de construir aprendizajes profundos y duraderos. 5,6

Desde la primera década del siglo XXI, se han utilizado diferentes medios para ayudar a la formación de personal de la salud que se encuentra a grandes distancias de los centros de simulación. Dentro de esta práctica, hemos visto el desarroIlo de términos como simulación remota, ${ }^{12,23}$ simulación en línea ${ }^{13,24}$ y telesimulación. ${ }^{14}$ Esta terminología relativamente nueva ha llevado a crear confusión entre los docentes que utilizan la simulación no presencial como estrategia de

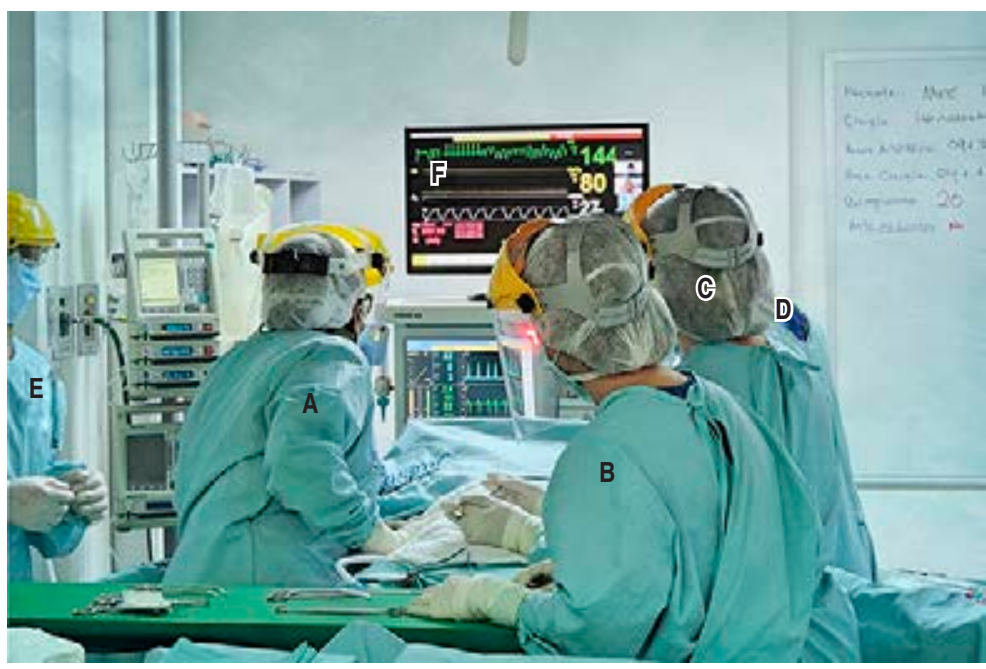

Figura 1: Telesimulación de crisis en el quirófano (vista del camarógrafo): A) confederado (cirujana), B) confederado (instrumentadora), C) confederado (ayudante), D) confederado (anestesiólogo), E) confederado (circulante), F) monitor de signos vitales con conexión a una plataforma de conferencias.

Fuente: los autores. enseñanza, aprendizaje y evaluación, por lo tanto, queremos mostrar aspectos que pueden ayudar a comprender mejor el alcance de cada uno de los conceptos más utilizados, cuyos limites pueden resultar borrosos (Tabla 1 ).

\section{EXPERIENCIA Y REFLEXIÓN: EVOLUCIÓN DE NUESTRO CONCEPTO}

En el primer trimestre de 2020 iniciamos simulación sincrónica en línea usando un formato muy similar al utilizado en nuestras prácticas docentes en la simulación de alta fidelidad presencial, esto es, orientación (briefing), caso simulado, el cual contaba con un director de escenario, confederado, paciente estandarizado y participantes, finalizando con un espacio de reflexión estructurada (debriefing). Lo anterior se realizaba transmitiendo a través de la red, compartiendo la pantalla de un monitor de signos vitales, imágenes diagnósticas y paraclínicos en una plataforma de reuniones (zoom $\left.{ }^{\circledR}\right)$. Utilizamos otras plataformas en el proceso; sin embargo, el retardo en la transmisión de audio y video nos llevó a rechazarlas, dado que el enganche cognitivo de los participantes se veía notablemente disminuido y nos alejaba de los objetivos.

Este modelo de simulación a distancia fue trabajado en pregrado de medicina y educación médica continua multidisciplinaria en el área de medicina intensiva y anestesiología. En cuanto a los resultados, los participantes lograron mejoría en el aspecto declarativo del conocimiento y alta satisfacción con la actividad simulada.

En el manejo de crisis en medicina perioperatoria dirigida únicamente a anestesiólogos, los congresos de la Sociedad de Anestesiología de Antioquia (SADEA) y de la Sociedad Colombiana

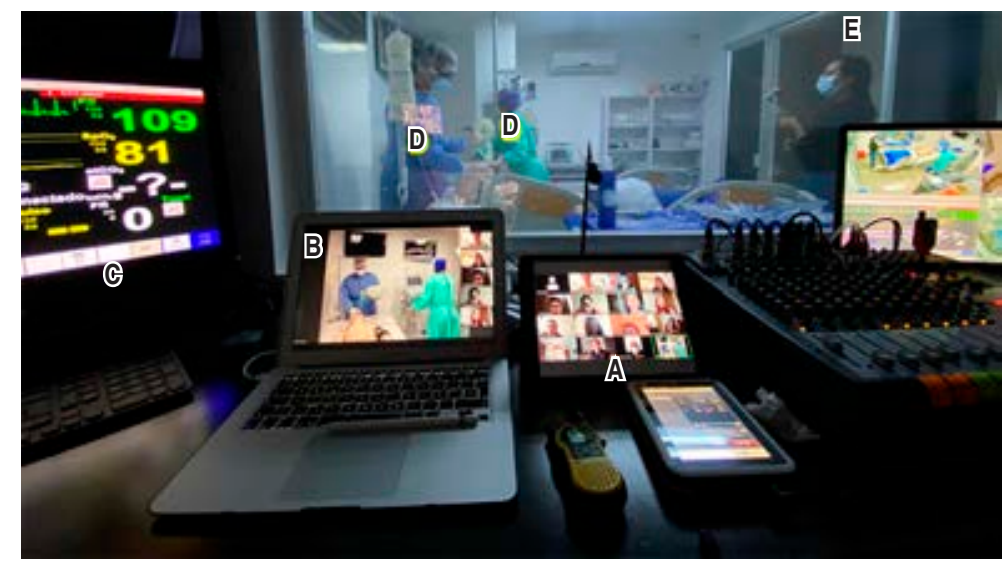

Figura 2:

Simulación en línea sincrónical telesimulación: A) vista del instructor (director del escenario), B) vista de los participantes destacada para todos por el anfitrión (vista del camarógrafo), C) monitor de signos vitales, $D$ ) confederados, E) camarógrafo. Fuente: los autores. 


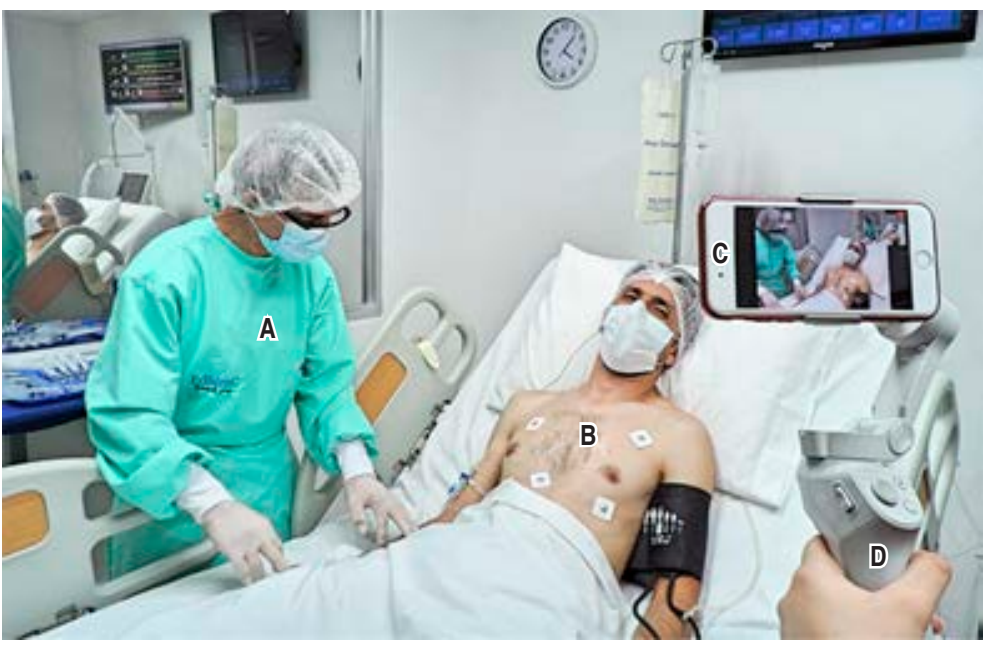

Figura 3: Elementos de la telesimulación: A) confederado, B) paciente estandarizado, C) teléfono móvil transmitiendo, D) estabilizador.

Fuente: los autores. de Anestesiología y Reanimación (S.C.A.R.E.) nos permitieron implementar algunas modificaciones sustanciales, como el retorno de los pacientes estandarizados y confederados al centro de simulación (Figura 1); desde allí transmitíamos un escenario a través de un dispositivo móvil conectado a la reunión, usando un estabilizador de imagen y un nuevo miembro en el equipo, el camarógrafo.

En este formato, los participantes se encuentran en sus casas, un equipo está en el centro de simulación; los participantes, siguiendo la estructura de la telemedicina, se encargan de brindar orientación y apoyo al equipo tratante que se encuentra, en este caso, simulando un caso de medicina perioperatoria (Figura 2).

Esta telesimulación fue trabajada inicialmente con casos de anafilaxia y vía aérea difícil; no obstante, a medida que se progresa en el dominio de una metodología, la complejidad de los escenarios es susceptible de aumentar. En la actualidad hacemos casos de medicina intensiva, trauma, reanimación, entre otros, en los que entrenamos especialmente sobre factores humanos y el aspecto declarativo de las habilidades técnicas. Encontramos un excelente enganche cognitivo de los profesionales participantes y una alta percepción de aprendizaje, que como ocurre en la simulación presencial, la explican desde la experiencia y el teledebriefing.

Consideramos que esta manera de hacer telesimulación resulta ser versátil, puede realizarse para entrenar cómo dar malas noticias, hasta ser empleada en escenarios complejos de crisis en quirófanos, unidad de terapia intensiva o escenarios extramurales. Puede utilizarse con maniquíes complejos o con pacientes estandarizados (Figura 3), todo depende del diseño curricular específico y de la experiencia del grupo docente.

\section{EVIDENCIA}

La evidencia empírica en este campo está en proceso de construcción, algunos trabajos con estudiantes de pregrado de medicina y residentes de anestesiología muestran mejoría en el dominio de conceptos relacionados a la atención segura de pacientes con COVID-19 y mejoría en el dominio de algunas habilidades no técnicas (comunicación, liderazgo, consciencia situacional), asimismo, satisfacción con la actividad simulada, percepción de aprendizaje y alta calidad del debriefing; no obstante, esta metodología también presenta limitaciones, como la dependencia de la calidad del internet, la dificultad de comunicación verbal simultánea por la saturación de los micrófonos, la imposibilidad de desarrollar habilidades motoras, a menos que el estudiante pueda tener acceso a un simulador, entrenar y recibir retroalimentación (sincrónica o asincrónica). 27-29

\section{RETOS}

La virtualización de la educación, presionada en gran medida por la pandemia, posiblemente ha sido mal comprendida. Si bien es cierto que en la actualidad los docentes usamos más las tecnológicas de la información y la comunicación dentro de nuestras prácticas pedagógicas (que es todo un logro), disponer de computadores y conexión a la red no garantiza per se la creación de entornos constructivos de aprendizaje, ya que si las actividades de formación se mantienen más en la enseñanza en cabeza del docente que en la experiencia reflexiva del aprendiz, terminan siendo prácticas anacrónicas que limitan el aprendizaje.

Parece claro que algunos de los aspectos más importantes en el aprendizaje son la motivación de quien aprende, ${ }^{30}$ su capacidad de comprometerse con la tarea (enganche cognitivo) ${ }^{31}$ y la dificultad de la tarea en sí misma, ${ }^{32,33}$ por lo tanto, un educador debe fijarse en estos aspectos a la hora de planear actividades docentes centradas en el aprendizaje, lo que incluye la simulación clínica, presencial y a distancia. ${ }^{34,35}$ En este último aspecto, dar su merecido lugar a la orientación (briefing), mantener escenarios simulados con car- 
ga cognitiva extrínseca muy baja (desde el diseño) y un tiempo dedicado a la reflexión apropiada convierte a la telesimulación en una actividad social y constructiva de aprendizaje.

\section{DISEÑO INSTRUCCIONAL DE TELESIMULACIÓN: RECOMENDACIONES PARA EDUCADORES}

El diseño instruccional requiere de un análisis metódico, para lo cual recomendamos contar con un equipo multidisciplinario con experiencia en educación y simulación. Existen varios modelos, nosotros usamos el modelo ADDIE (Análisis, Diseño, Desarrollo, Implementación, Evaluación), ${ }^{36,37}$ adaptándolo a la simulación a distancia y manteniendo una estructura similar a la simulación presencial. ${ }^{5}$

Análisis: es el punto de inicio, por lo tanto, fundamental en el proceso. En esta fase se identifica y delimita el problema, se analizan las necesidades de formación y se ajusta a la población (participantes). En medicina perioperatoria, es esencial contemplar a todos los miembros del equipo, pues los objetivos de aprendizaje no son necesariamente los mismos para todos. Recomendamos plantear dos objetivos de conocimiento teórico/técnico y dos de factores humanos.

Diseño: en esta fase se toman las necesidades de formación y los objetivos de aprendizaje para establecer cuál es la mejor forma de realizar la instrucción. Se debe escribir según el público al que va dirigida la formación, lo ideal es establecer sus perfiles, posibles saberes previos, etcétera.

Desarrollo: con los datos de la fase anterior, se organiza la actividad simulada en línea, se crean los escenarios, se decide qué recursos movilizar (calidad de internet, cámaras, monitores, confederados, pacientes estandarizados, simuladores, flujogramas de simulación, bibliografía a entregar, instructores, etcétera). Recomendamos hacer siempre un piloto de cada escenario simulado para asegurar la calidad y minimizar fallos (que pueden ser frecuentes al principio).

Implementación: es la realización de la actividad de instrucción; en esta fase recomendamos hacer una muy buena introducción (briefing), conocer a los participantes, ofrecerles nuestra ayuda para alcanzar sus objetivos de aprendizaje, despejar sus dudas, informar las fortalezas y debilidades que tiene la simulación a distancia, explicar qué esperamos de ellos y qué haremos. ${ }^{38}$ Si lo hacemos bien, es muy probable que los participantes tengan un adecuado enganche cognitivo con la actividad y que participen activamente durante la reflexión posterior al evento (debriefing).

En cuanto al desarrollo del caso, para que sea eficiente, debe contar con congruencia en el argumento, en los elementos del escenario y en la conducta del equipo de simulación, cuidando el tiempo de simulación, pues suele ser mayor que en simulación presencial, debido a los turnos en el habla de los participantes. De esta manera, el participante tendrá mayor posibilidad de experimentar realismo.

Evaluación: el objetivo de la última fase es determinar el impacto de la actividad formativa. Nosotros acostumbramos usar el modelo de cuatro niveles de Kirkpatrick ${ }^{39}$ (nivel 1: satisfacción; nivel 2: aprendizaje; nivel 3: transferencia; nivel 4: impacto en la salud de los pacientes). En el caso de la telesimulación, para abordar los primeros dos niveles, recomendamos usar el debriefing estructurado como evaluación formativa. ${ }^{6}$ El debriefing es un componente central de la simulación tanto presencial como en línea, que permite establecer las interacciones, el proceso y los desenlaces del caso; ${ }^{40}$ ayuda a comprender las representaciones internas (modelos mentales) de los participantes facilitando así el aprendizaje profundo y duradero. Si se requiere certificar la aprobación, se debe realizar una evaluación sumativa, recomendamos que sea con rúbricas, idealmente validadas.

\section{CONCLUSIONES}

La pandemia por COVID-19 ha traído grandes cambios en muchas de las esferas de la vida diaria, y la académica es una de ellas con la educación en línea. En este sentido, la formación en medicina perioperatoria puede realizarse con ayuda de las tecnologías de la información y la comunicación en conjunto con las tecnologías de simulación disponibles en los centros de simulación.

La telesimulación es una estrategia versátil, eficiente y de relativo bajo costo, que permite enseñar, aprender y evaluar en estos tiempos de pandemia y en el futuro inmediato, con las ventajas que ofrece la reflexión intencionada en tiempo real, lo que redunda en mayor enganche cognitivo y posiblemente mayor aprendizaje.

La telesimulación tiene algunas limitaciones como la conectividad a internet y requiere ser complementada con simulación presencial para el entrenamiento de habilidades motoras y el reforzamiento de habilidades no técnicas. 


\section{AGRADECIMIENTOS}

A todos los profesionales y técnicos de VitalCare y de la Universidad Cooperativa de Colombia que participaron en la realización del taller precongreso en línea de SADEA 2020 y en el taller de manejo de crisis del congreso nacional S.C.A.R.E 2021.

\section{REFERENCIAS}

1. Cooper JB, Taqueti VR. A brief history of the development of mannequin simulators for clinical education and training. Postgrad Med J. 2008; 84 (997): 563-570.

2. Maestre J, Palazuelos J, del Moral I, Simon R. La simulación clínica como herramienta para facilitar el cambio de cultura en las organizaciones de salud: aplicación práctica de la teoría avanzada del aprendizaje. Rev Colomb Anestesiol. 2014; 42 (2): 124-128.

3. Gaba DM. Crisis resource management and teamwork training in anaesthesia. $\mathrm{Br}$ J Anaesth. 2010; 105 (1): 3-6.

4. Dieckmann P, Gaba D, Rall M. Deepening the theoretical foundations of patient simulation as social practice. Simul Healthc. 2007; 2 (3): 183-193.

5. Díaz-Guio DA, Ruiz-Ortega FJ. Relationship among mental models, theories of change, and metacognition : structured clinical simulation. Colomb J Anesthesiol. 2019; 47 (14): 113-6. Available in: http://dx.doi. org/10.1097/CJ9.0000000000000107

6. Rudolph JW, Simon R, Raemer DB, Eppich WJ. Debriefing as formative assessment: closing performance gaps in medical education. Acad Emerg Med. 2008; 15 (11): 1010-1016.

7. Flin R, Patey R, Glavin R, Maran N. Anaesthetists' nontechnical skills. Br J Anaesth. 2010; 105 (1): 38-44.

8. Gaba DM, Howard SK, Flanagan B, Smith BE, Fish KJ, Botney R. Assessment of clinical performance during simulated crises using both technical and behavioral ratings. Anesthesiology. 1998; 89 (1): 8-18.

9. Zhu N, Zhang D, Wang W, Li X, Yang B, Song J, et al. A Novel Coronavirus from Patients with Pneumonia in China, 2019. N Engl J Med. 2020; 382 (8): 727-733.

10. Li, C; Lalani F. The COVID-19 pandemic has changed education forever. This is how. World Economic Forum. 2020. Available in: https://www.weforum. org/agenda/2020/04/coronavirus-education-globalcovid19-online-digital-learning/

11. UNESCO. COVID-19 Educational Disruption and Response. 2020 [cited 2020 Aug 11]. Available in: https://en.unesco.org/covid19/educationresponse

12. Burckett-St, Laurent DA, Niazi A, Cunningham MS, Jaeger M, Abbas S, et al. A valid and reliable assessment tool for remote simulation-based ultrasound-guided regional anesthesia. Reg Anesth Pain Med. 2014; 39 (6): 496-501.

13. Cant RP, Cooper SJ. Simulation in the Internet age: The place of Web-based simulation in nursing education: An integrative review. Nurse Educ Today. 2014; 34 (12): 1435-1442. Available in: http://dx.doi. org/10.1016/j.nedt.2014.08.001
14. McCoy CE, Sayegh J, Alrabah R, Yarris LM. Telesimulation: an innovative tool for health professions education. AEM Educ Train. 2017; 1 (2): 132-136.

15. King AB, Alvis BD, McEvoy MD. Enhanced recovery after surgery, perioperative medicine, and the perioperative surgical home: Current state and future implications for education and training. Curr Opin Anaesthesiol. 2016; 29 (6): 727-732.

16. Martín Delgado MC, Gordo Vidal F. Medicina intensiva perioperatoria. Med Intensiva. 2019; 43 (7): 427-34.

17. Vasco-Ramírez M. Are anesthesiologists ready for the "new normal"? Colomb J Anesthesiol. 2020; 48 (4): e921. Available in: https://doi.org/10.5554/22562087.e921

18. Díaz-Guio D, Ospina-vélez J, Ricardo-zapata A. COVID-19: una crisis que requiere medidas de formación urgentes. Simulación Clínica. 2020; 2 (1): 6-8.

19. Díaz-Guio DA, Díaz-Guio Y, Pinzón-Rodas V, DíazGomez AS, Guarín-Medina JA, Chaparro-Zúñiga $Y$, et al. COVID-19: biosafety in the intensive care unit. Curr Trop Med reports. 2020; 7: 104-11. Available in: https://doi.org/10.1007/s40475-020-00208-z

20. Díaz-Guio D, Ricardo-Zapata A, Ospina-Velez J, Gómez-Candamil G, Mora-Martinez S, RodriguezMorales A. Cognitive load and performance of health care professionals in donning and doffing PPE before and after a simulation-based educational intervention and its implications during the COVID-19 pandemic for biosafety. Infez Med. 2020; 28 (Suppl 1):111117. Available in: https://pubmed.ncbi.nlm.nih. gov/32532947/

21. Díaz-Guio DA, Ríos-Barrientos E, Santillán-Roldan PA, Medina V; Salazar-Ocampo DF, Cimadevilla-Calvo B, Ricardo-Zapata A. Factores humanos y seguridad del personal de salud en tiempos de pandemia. Simulación Clínica. 2020; 2 (2): 81-85.

22. Steadman RH, Huang YM. Simulation for quality assurance in training, credentialing and maintenance of certification. Best Pract Res Clin Anaesthesiol. 2012; 26 (1): 3-15.

23. LeFlore JL, Sansoucie DA, Cason CL, Aaron A, Thomas PE, Anderson M. Remote-controlled distance simulation assessing neonatal provider competence: A feasibility testing. Clin Simul Nurs. 2014; 10 (8): 419-424. Available in: http://dx.doi.org/10.1016/j. ecns.2014.04.004

24. Huun K. Virtual Simulations in Online Nursing Education: align with quality matters. Clin Simul Nurs. 2018; 22: 26-31. Available in: https://doi. org/10.1016/j.ecns.2018.07.002

25. Jewer J, Parsons MH, Dunne C, Smith A, Dubrowski A. Evaluation of a mobile telesimulation unit to train rural and remote practitioners on high-acuity lowoccurrence procedures: Pilot randomized controlled trial. J Med Internet Res. 2019; 21 (8):1-17.

26. Dubovi I. Online Computer-Based Clinical Simulations: The role of visualizations. Clin Simul Nurs. 2019; 33: 35-41.

27. Patel SM, Miller CR, Schiavi A, Toy S, Schwengel DA. The sim must go on: adapting resident education to the COVID-19 pandemic using telesimulation. Adv Simul. 2020; 5 (1):1-11.

28. McCoy CE, Sayegh J, Rahman A, Landgorf M, Anderson C, Lotfipour S. Prospective randomized crossover 
study of telesimulation versus standard simulation for teaching medical students the management of critically III patients. AEM Educ Train. 2017; 1 (4): 287-292.

29. Díaz-Guio DA, Ríos-Barrientos E, Santillán-Roldan PA, Díaz-Gómez AS, Ricardo-Zapata A, Mora-Martinez S, et al. Online-synchronized clinical simulation: an efficient teaching-learning option for the COVID-19 pandemic time and beyond. Adv Simul. 2021; 6: 30. Available in: https://doi.org/10.21203/rs.3.rs-106185/v1

30. Vansteenkiste M, Sierens E, Soenens B, Luyckx K, Lens W. Motivational Profiles From a Self-Determination Perspective: The quality of motivation matters. J Educ Psychol. 2009; 101 (3): 671-688.

31. Chi MTH, Wylie R. The ICAP Framework: Linking cognitive engagement to active learning outcomes. Educ Psychol. 2014; 49 (4): 219-243.

32. Sweller J. Cognitive load theory, learning difficulty, and instructional design. Learn Instr. 1994; 4 (4): 295-312.

33. Fraser K, Ma I, Teteris E, Baxter H, Wright B, Mclaughlin K. Emotion, cognitive load and learning outcomes during simulation training. Med Educ. 2012; 46 (11): 1055-1062.

34. Taylor DCM, Hamdy H. Adult learning theories: Implications for learning and teaching in medical education: AMEE Guide No. 83. Med Teach. 2013; 35 (11).

35. Fraser KL, Ayres P, Sweller J. Cognitive load theory for the design of medical simulations. Simul Healthc. 2015;10 (5): 295-307.
36. Dick, W; Carey L. The Systematic Design of Instruction. 4th ed. New York: Harper Collins College Publishers; 1996.

37. Patel SR, Margolies PJ, Covell NH, Lipscomb C, Dixon LB. Using Instructional Design, Analyze, Design, Develop, Implement, and Evaluate, to Develop e-Learning Modules to Disseminate Supported Employment for Community Behavioral Health Treatment Programs in New York State. Front Public Heal. 2018; 6: 113.

38. Rudolph JW, Raemer DB, Simon R. Establishing a safe container for learning in simulation the role of the presimulation briefing. Simul Healthc. 2014; 9 (6): 339-349.

39. Kirkpatrick, JD; Kirkpatrick W. Kirkpatrick's four levels of training evaluation. Alexandria: ATD Press; 2016.

40. Martinelli SM, Chen F, Isaak RS, Huffmyer JL, Neves SE, Mitchell JD. Educating Anesthesiologists During the Coronavirus 2019 Pandemic and Beyond. Anesth Analg. 2021; 132 (3): 585-593. Available in: https:// pubmed.ncbi.nlm.nih.gov/33201006/

\section{Correspondencia:}

Diego Andrés Díaz-Guio

E-mail: andres.diaz@vitalcare.co 